\title{
Aspectos clínico-epidemiológicos da infecção puerperal em maternidade de referência no Amazonas de 2018 a 2019
}

\author{
Clinical-epidemiological aspects of puerperal infection in reference maternity in the \\ Amazonas from 2018 to 2019
}

\section{Aspectos clínico-epidemiológicos de la infección puerperal en maternidad de referencia en las Amazonas desde 2018 a 2019}

Maria do Perpétuo Socorro Mota Marinho ${ }^{1 *}$, Claudia Marques de Oliveira Soeiro.

\begin{abstract}
RESUMO
Objetivo: Avaliar a prevalência da infecção puerperal em uma maternidade pública no Amazonas, os fatores de risco associados e a relação entre esquemas de antibioticoterapia mais adotados e os desfechos frente à infecção puerperal. Métodos: Estudo descritivo e retrospectivo, realizado no período de janeiro de 2018 a dezembro de 2019 com base na análise das informações contidas em prontuários das pacientes que foram submetidas a parto vaginal ou cesárea e apresentaram infecção puerperal no período citado. Resultados: $A$ amostra foi 351 gestantes, com prevalência de infecção puerperal de 2,28\% durante o período estudado. A média de idade das pacientes foi 23,9 anos. A indicação mais prevalente para a cesariana foi Sofrimento Fetal Agudo (21,7\%). A antibioticoprofilaxia adequada foi administrada em $32,5 \%$, sendo realizada com Cefalotina em 59,6\% da amostra, seguida da Cefazolina em 40,3\%. Para o tratamento, o esquema mais utilizado foi o tríplice Cefalotina associada a Gentamicina e Metronidazol (53,8\%). A ressutura foi realizada em um terço das pacientes $(33,1 \%)$. Conclusão: as complicações na fase do puerpério se destacam e se mostram cada vez mais presentes, e as medidas de controle de infecção puerperal requerem educação adequada, melhorias de protocolos e introdução de novas diretrizes clínicas.
\end{abstract}

Palavras-chave: Infecção puerperal, Cesárea, Sepse, Mortalidade materna.

\begin{abstract}
Objective: To evaluate the prevalence of puerperal infection in a public maternity hospital in Amazonas, the associated risk factors and the relationship between the most adopted antibiotic therapy regimens and the outcomes of puerperal infection. Methods: Descriptive and retrospective study, carried out from January 2018 to December 2019, based on the analysis of information contained in the medical records of patients who underwent vaginal or cesarean delivery and dissipated puerperal in the aforementioned period. Results: The sample consisted of 351 pregnant women, with a prevalence of puerperal infection of $2.28 \%$ during the study period. The mean age of patients was 23.9 years. The most prevalent indication for cesarean section was Acute Fetal Suffering (21.7\%). Appropriate antibiotic prophylaxis was administered in $32.5 \%$, being performed with Cephalotin in $59.6 \%$ of the sample, followed by Cefazolin in $40.3 \%$. For treatment, the most used regimen was the triple cephalothin associated with gentamicin and metronidazole (53.8\%). Resuture was performed in one third of patients (33.1\%). Conclusion: complications in the puerperium stage stand out and are increasingly present, and puerperal infection control measures are adequate education, process improvements and introduction of new clinical guidelines.
\end{abstract}

Keywords: Puerperal infection, Cesarean section, Sepsis, Maternal mortality.

\section{RESUMEN}

Objetivo: Evaluar la prevalencia de infección puerperal en una maternidad pública de Amazonas, los factores de riesgo asociados y la relación entre los regímenes de antibioticoterapia más adoptados y los resultados de la infección puerperal. Métodos: Estudio descriptivo y retrospectivo, realizado de enero de 2018 a diciembre de 2019, con base en el análisis de la información contenida en las historias clínicas de pacientes que fueron sometidas a parto vaginal o cesárea y puerperal disipado en el período mencionado. Resultados: La muestra estuvo conformada por 351 gestantes, con una prevalencia de infección puerperal de $2.28 \%$ durante el período de estudio. La edad media de los pacientes fue de 23,9 años. La indicación más prevalente de cesárea fue Sufrimiento Fetal Agudo (21,7\%). Se administró profilaxis antibiótica adecuada en el 32,5\%, realizándose

1 Universidade do Estado do Amazonas (UEA), Manaus - AM. *E-mail: cmosoeiro@hotmail.com 
con cefalotina en el $59,6 \%$ de la muestra, seguida de cefazolina en el 40,3\%. Para el tratamiento, la pauta más utilizada fue la triple cefalotina asociada a gentamicina y metronidazol (53,8\%). La reanimación se realizó en un tercio de los pacientes $(33,1 \%)$. Conclusión: las complicaciones en la etapa del puerperio se destacan y están cada vez más presentes, y las medidas de control de la infección puerperal son la educación adecuada, la mejora de los procesos y la introducción de nuevas guías clínicas.

Palabras clave: Infección puerperal, Cesárea, Sepsis, Mortalidad materna.

\section{INTRODUÇÃO}

A infecção puerperal é definida como a que se origina no aparelho genital após parto recente, correspondendo a $3^{\mathrm{a}}$ maior causa de mortalidade materna no Brasil (UNIVERSIDADE FEDERAL DO RIO DE JANEIRO (UFRJ), 2018). De uma forma mais abrangente, a infecção puerperal pode ser conceituada como um processo infecciosos pós-parto, seja por causas genitais, como infecções no útero, anexos e sítio cirúrgico, ou por causas extragenitais, como, por exemplo, mastite, tromboflebite, complicações respiratórias e infecções do trato urinário (REIS AT, 2012).

De acordo com o estudo de Martins ACS e Silva LS (2018), a infecção puerperal foi a quinta maior causa de mortalidade materna nos anos de 2000 a 2009 no Brasil, representando 5,12\%. Hipertensão e a hemorragia são as principais causas de morte materna no Brasil são. Ademais, a infecção puerperal e o aborto também são destacados como importantes causas obstétricas diretas de morte materna.

A maioria das infecções puerperais ocorre após a alta hospitalar, o que determina problema em sua notificação e dificuldade de mensurar sua real incidência. São poucos os trabalhos publicados no Brasil que tratam desse tema. Sobretudo em suas regiões menos desenvolvidas, onde as condições socioeconômicas desfavoráveis ainda contribuem para a inadequada assistência ao parto e puerpério, principalmente em cidades do interior, infecções graves durante esse período continuam prevalentes e destacam-se como principais causas de morte materna (GONÇALVES MVC, et al., 2012).

O diagnóstico da infecção puerperal, em termos gerais, é dado através da temperatura maior ou igual a $38^{\circ} \mathrm{C}$, com tempo médio de duração maior que quarenta e oito horas, que aparece nos primeiros dez dias após o parto, excluindo-se as primeiras vinte e quatro horas. São exceções a esta regra as puérperas com infeção em ferida operatória, como episiotomia ou cesariana, pois estas frequentemente evoluem com quadro afebril (Agência Nacional de Vigilância Sanitária (ANVISA), 2017).

Sobre os exames complementares, a avaliação laboratorial pode ajudar a identificar o agente etiológico, origem e gravidade do caso. Alguns exemplos de exames úteis são: hemograma, exame sumário de urina, hemocultura, urinocultura e cultura de secreções suspeitas, além de radiografia de tórax e ultrassonografia de partes moles, para investigação de abscessos (UFRJ, 2018).

Os fatores de risco associados à Infecção Puerperal antes do parto (período pré-natal) são as infecções maternas, em especial as do trato urinário; comorbidades maternas, como anemia, diabetes, obesidade, imunossupressão; condições sociais desfavoráveis; ausência ou baixa qualidade da assistência pré-natal; infecção cérvico-vaginal por Chlamydia trachomatis e Trichomonas vaginalis; vaginoses bacterianas, principalmente por bactérias anaeróbias, como Gardnerella vaginalis, Bacteroides sp., Mobiluncus sp., Mycoplasma hominis, e infecções sexualmente transmissíveis, como Gonorreia, Sífilis e infecção provocada pelo Vírus da Imunodeficiência Humana (HIV) (ANVISA, 2017).

Dados coletados do Sistema Único de Saúde do Brasil demonstram que o risco de infecção puerperal na população materna é em geral 4,35 vezes maior em comparação com outros países, e que a cesariana é um fator importante para a mortalidade materna por infecção puerperal, já que o risco para tal é 3 vezes mais elevada do que para o parto vaginal. Segundo um artigo publicado por Say L, et al. (2014), o Brasil é o segundo país com maior taxa de cesáreas, perdendo apenas para República Dominicana, e o aumento de tal prática se transformou em uma "epidemia". A World Health Organization (WHO, 2015) recomenda para comunidade internacional de saúde que a taxa ideal de cesáreas deve ficar entre $10 \%$ e $15 \%$ de todos os partos realizados. O Brasil apresenta média de $55 \%$ de taxa média de cesáreas, mais que o triplo da margem recomendada pela Organização Mundial da Saúde. 
O grande número de partos cesarianos tem uma grande parcela de contribuição para a elevada taxa de infecção puerperal do país, pois a cesariana apresenta maior risco de desenvolver infecção puerperal em relação aos partos naturais (ANVISA, 2017).

Em relação aos fatores de risco para infecção de ferida operatória, os comumente discutidos na literatura são o tempo prolongado de cirurgia - o risco para o desenvolvimento da infecção é proporcional ao tempo de cirurgia; lesões acidentais de tecidos e órgãos; necessidade de realização de cesariana de emergência; além da falta ou incorreta administração de antibióticos profiláticos (ANVISA, 2017).

De acordo com Dias CMCC e Oliveira JR (2011), os índices de endometrite pós-parto cesárea podem ser reduzidas em aproximadamente $50 \%$ com o uso da antibioticoprofilaxia. No entanto, as literaturas atuais questionam se a profilaxia deve ser realizada em todas as mulheres, além de qual a droga mais apropriada e quando iniciar a sua administração. Nos dias de hoje, a classe de antibióticos mais utilizados são as cefalosporinas, administrando-se geralmente em dose única no pré-operatório ou após clampear o cordão umbilical.

A antibioticoprofilaxia preconizada em diversas literaturas consiste no uso de cefalosporina de primeira geração, ou seja, Cefazolina $2 \mathrm{~g}$ até $120 \mathrm{~kg}$ e $3 \mathrm{~g}$ acima de $120 \mathrm{~kg}$, em dose única, administrada nos 60 minutos que antecedem a incisão. Em caso de duração da cirurgia maior que 4 horas e/ou sangramento maior que $1,5 \mathrm{~L}$, nova dose deve ser administrada posteriormente. Pacientes alérgicas a cefalosporinas, ou com reação anafilática grave à penicilina devem utilizar clindamicina $900 \mathrm{mg}$ com ou sem aminoglicosídeo (Gentamicina $5 \mathrm{mg} / \mathrm{kg}$ ) (WHO, 2015; AMERICAN COLLEGE OF OBSTETRICIANS AND GYNECOLOGISTS (ACOG), 2011).

Em um estudo realizado no Amazonas por Praia IG e Silva SM (2021) com 303 gestantes que apresentaram infecção de ferida operatória, observou-se que $87 \%$ das pacientes haviam recebido antibiotiprofilaxia com cefalosporinas de primeira geração. Desta forma, percebe-se que antibioticoprofilaxia não representa proteção total contra a infecção de ferida operatória, pois diversos outros fatores podem interferir para o surgimento do quadro infeccioso, como explanado anteriormente.

Dessa forma, foi possível verificar que os trabalhos científicos sobre fatores de risco para infecção puerperal são escassos e pouco atualizados, devido, entre outros fatores, à subnotificação dos casos no país. Cabe salientar que o Brasil apresenta taxa de mortalidade materna elevada, o triplo do limite estabelecido para cesárea pela Organização Munidial da Saúde (OMS) (2015).

Em detrimento da importância da infecção puerperal na morbimortalidade materna e a elevada incidência na região Norte do Brasil, torna-se necessário conhecer os aspectos epidemiológicos da infecção puerperal em uma maternidade no Amazonas, visando a redução de casos. O objetivo deste estudo consiste em avaliar a prevalência de infecção puerperal no período de janeiro de 2018 a dezembro de 2019, além de descrever os principais fatores associados à infecção.

\section{MÉTODOS}

Trata-se de um estudo descritivo e retrospectivo, com base na análise das informações contidas em prontuários das pacientes que foram submetidas ao parto vaginal e cesárea e apresentaram infecção puerperal no período de janeiro de 2018 a dezembro de 2019 em uma maternidade pública de referência no Amazonas.

Os critérios de inclusão do estudo foram pacientes que foram submetidas ao parto vaginal ou cesárea com diagnóstico de infecção puerperal no período de janeiro de 2018 a dezembro de 2019. Foram excluídos prontuários cujos dados necessários para análise estavam incompletos ou inelegíveis; pacientes transferidos ou evadidos durante o tratamento da infecção puerperal; pacientes que foram à óbito durante o tratamento por outras causas descritas no prontuário.

Os dados foram colhidos através de uma ficha de coleta no setor Serviço de Arquivamento Médico e Estatística (SAME). Foram catalogados os dados referentes à idade, comorbidades maternas, completude do 
pré-natal, infecção prévia a cirurgia, via de parto, sítio da infecção puerperal, além dos esquemas e tempo de administração da antibioticoterapia prescrita, além de outros dados necessários compatíveis com os objetivos específicos. As variáveis escolhidas são compatíveis com os principais fatores de risco associados a infecções puerperais explanados pela literatura científica e que estão descritas nos prontuários analisados.

Ao final desta análise, 380 prontuários de infecção puerperal foram analisados, no entanto a amostra final deste estudo consistiu em 351 puérperas que se adequaram aos critérios de inclusão. Os demais prontuários restantes (29) não contemplavam os dados requeridos pelo projeto, sendo, pois, excluídos deste estudo para análise das variáveis. O projeto foi submetido e aprovado pelo Comitê de Ética em Pesquisa (CEP) através da Plataforma Brasil, com o CAEE 46215321.4.0000.5016.

\section{RESULTADOS}

Foram realizados 7086 partos cesáreos e 9546 partos vaginais nos anos em estudo, com ou sem infecção puerperal. As taxas de realização de cesarianas nos anos de 2018 e 2019 em uma maternidade pública de referência no Amazonas foram respectivamente: $42 \%$ e $43,2 \%$, já as de parto normal foram $58 \%$ no ano de 2018 e 56,8\% em 2019. Quanto à taxa global de infecção puerperal, nesses 2 anos, foi de 2,28\%, uma vez que do total de 16632 partos, 380 evoluíram com Infecção Puerperal (IP).

De todo o universo de pacientes com IP, foram analisados 380 prontuários de pacientes que evoluíram com IP no período de janeiro de 2018 a dezembro de 2019. Ao final desta análise, a amostra deste estudo consistiu em 351 puérperas que foram submetidas a partos cesáreos ou normais, foram diagnosticadas com IP e que se adequaram aos critérios do estudo.

A via de parto cirúrgica, ou seja, cesárea, foi predominante nas pacientes que desenvolveram Infecção Puerperal, constituindo $96,6 \%(n=339)$ da amostra. A via vaginal, por conseguinte, caracterizou $3,4 \%(n=12)$ da amostra. A maioria das pacientes (a moda) do estudo apresentava intervalo de idade entre 20-24 anos (28,4\%, n=103), com a média de 23,9 anos, desvio padrão de 16,56 anos e mediana de 24 anos. Os extremos de idade nesta casuística foram representados somente por 7 pacientes (1,99\% da amostra) com menos de 15 anos e 13 pacientes $(3,7 \%)$ com mais de 39 anos.

Em relação à procedência das pacientes com infecção puerperal, a maior parte da população em estudo $(84,6 \%, n=297)$ provém da cidade de Manaus, capital do Amazonas.

Ao fazer a análise do tempo de hospitalização das pacientes, observa-se que a maior parte das pacientes $(35,8 \%, n=126)$ foram internadas por um curto período de tempo, entre 1 e 5 dias, seguidas pelo grupo das que foram internadas por 6 a 10 dias $(23,9 \%, n=84)$ e por 11 a 15 dias $(18,2 \%, n=64)$, respectivamente (Gráfico 1).

Gráfico 1 - Tempo de Hospitalização das pacientes com infecção.

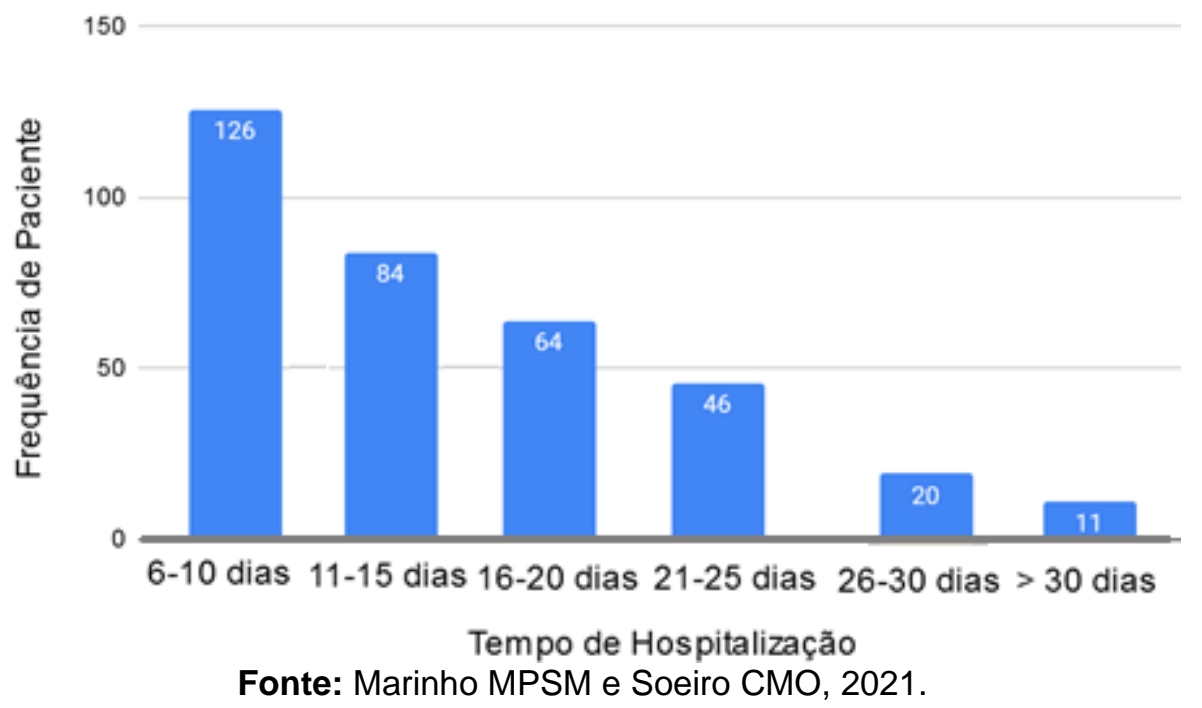


Em relação à paridade das pacientes acometidas de infecção puerperal, 38,7\% ( $n=136)$ apresentaram a infecção puerperal na primeira gestação, secundigesta $19,7 \%(n=69)$, seguida das multíparas com $41,6 \%$ $(n=146)$.

Sobre o pré-natal, 68,9\% $(\mathrm{n}=242)$ das pacientes o realizaram, independente do número de consultas. Entretanto, 21,9\% $(n=77)$ não realizarem sequer 1 consulta de pré-natal. Em relação às comorbidades associadas, $51 \%(n=179)$ das pacientes apresentavam alguma destas, notou-se que o mais prevalente foi a Infecção do Trato Urinário, presente em 37,6\% $(n=132)$ das pacientes. Em seguida temos a Síndrome Hipertensiva, presente em 5,4\% $(n=19)$ das mesmas, seja ela pré-eclâmpsia/eclâmpsia, hipertensão crônica, pré-eclâmpsia sobreposta à hipertensão crônica e hipertensão gestacional. A seguir estão descritos os dados sobre a realização de pré-natal e as comorbidades mais frequentes encontradas nas pacientes do universo de estudo (Tabela 1).

Tabela 1 - Análise clínico-epidemiológica das pacientes que tiveram infecção puerperal.

\begin{tabular}{lcc}
\hline Variáveis & $\mathbf{N}$ & $\mathbf{1}$ \\
\hline Total de prontuários analisados & $\mathbf{3 5 1}$ & $\mathbf{1 0 0 \%}$ \\
\hline Pré-natal & & $68,9 \%$ \\
\hline Sim & 242 & $21,9 \%$ \\
Não & 77 & $9,1 \%$ \\
Não Informado & 32 & $0,28 \%$ \\
\hline Comorbidades & & $1,4 \%$ \\
\hline Anemia & 1 & $37,6 \%$ \\
Diabetes Mellitus & 5 & $0,9 \%$ \\
Infecção do Trato Urinário & 132 & $1,7 \%$ \\
Infecção por HIV & 3 & $2,6 \%$ \\
Leucorreia & 6 & $1,1 \%$ \\
Obesidade & 9 & $5,4 \%$ \\
Sífilis & 4 & \\
Síndrome Hipertensiva & 19 &
\end{tabular}

Fonte: Marinho MPSM e Soeiro CMO, 2021.

Dentre a amostra de pacientes que realizaram pré-natal, apenas 5,8\% $(n=14)$ das pacientes realizaram 1 consulta, 10,3\% ( $n=25) 2$ consultas, $7,4 \%(n=18) 3$ consultas, $12,4 \%(n=30) 4$ consultas, 11,2\% ( $n=27) 5$ consultas e $52,9 \%(n=128)$ tiveram seis ou mais consultas. A média de consultas no pré-natal foi de 5,76 consultas, com desvio padrão de 2,94 consultas (Gráfico 2).

Gráfico 2 - Frequência de consultas no Pré-natal.

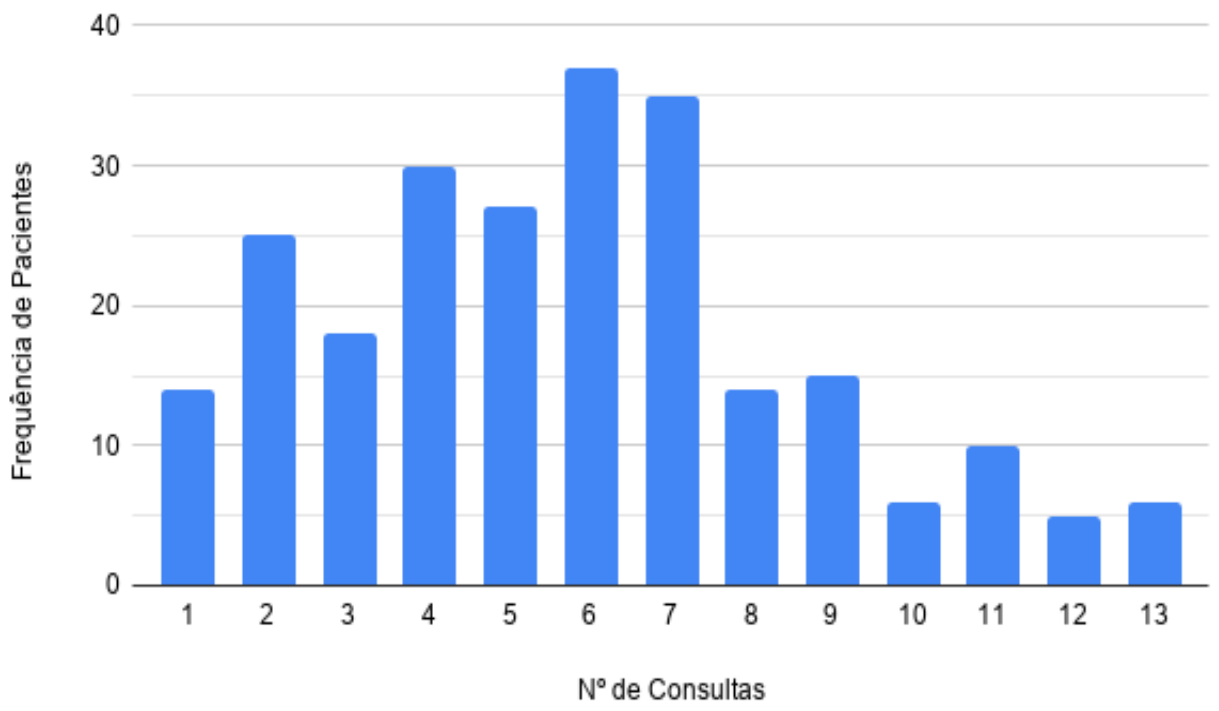

Fonte: Marinho MPSM e Soeiro CMO, 2021. 
As indicações clínicas e obstétricas que levaram à realização da cesárea das pacientes desta casuística estão descritas a seguir. Algumas tiveram mais de uma indicação, portanto o número de indicações não coincide com o total de prontuários estudados. Além disso, algumas pacientes já haviam recebido alta e retornaram à maternidade queixando-se dos comemorativos de IP, por conseguinte, na oportunidade, não discriminaram a indicação para o parto cirúrgico em suas fichas de atendimento (Tabela 2).

Pode-se verificar que a indicação mais frequente foi o Sofrimento Fetal, com $21,7 \%$ dos casos ( $n=76$ ); seguida da Desproporção Cefalopélvica, que predominou em 20,5\% dos casos $(n=72)$ e Parada de Progressão com $8 \%$ dos casos $(n=28)$. As indicações Gemelaridade, Centralização Fetal, Situação Transversa e Projeto Nascer estão enquadradas em "outros" e representam, juntas, 1,7\%, de acordo com a tabela abaixo.

Tabela 2 - Indicações clínicas e/ou obstétricas que evoluíram para realização da cesárea.

\begin{tabular}{lcc}
\hline Indicações para o Parto Cesáreo & N & $\%$ \\
\hline Amniorrexe Prematura & 20 & $5,7 \%$ \\
Apresentação Pélvica & 4 & $1,1 \%$ \\
Desproporção Cefalopélvica & 72 & $20,5 \%$ \\
Distúrbios Hipertensivos da Gravidez & 24 & $6,8 \%$ \\
Iteratividade & 4 & $1,1 \%$ \\
Macrossomia & 4 & $1,1 \%$ \\
Oligoidrâmnio & 16 & $4,6 \%$ \\
Parada de Progressão & 28 & $8 \%$ \\
Polidrâmnio & 4 & $1,1 \%$ \\
Pós-datismo & 16 & $4,6 \%$ \\
Prematuridade & 4 & $1,1 \%$ \\
Rotura Prematura das Membranas Amnióticas & 4 & $1,1 \%$ \\
Sofrimento Fetal & 76 & 21,7 \\
Outras com frequência inferior a 1\% & 6 & $1,7 \%$ \\
\hline
\end{tabular}

Fonte: Marinho MPSM e Soeiro CMO, 2021.

Verificou-se que a antibioticoprofilaxia foi realizada em 32,5\% ( $n=114)$ das pacientes que apresentaram posteriormente infecção da ferida operatória, sendo que $13,7 \%(n=48)$ não realizaram antibioticoprofilaxia e em 53,8\% ( $n=189)$ esta informação não constava em prontuário. A classe utilizada na profilaxia foi a cefalosporina de primeira geração, dentro da qual, o esquema mais utilizado foi a Cefalotina, representando $59,6 \%(n=68)$ dos casos, seguido da Cefazolina, em 40,3\% ( $n=46)$ dos casos, administrada na indução anestésica (Tabela 3).

Tabela 3 - Perfil da antibioticoprofilaxia utilizada nas pacientes que evoluíram para infecção de sítio cirúrgico após cesárea.

\begin{tabular}{lcc}
\hline Variáveis & $\mathbf{N}$ & $\%$ \\
\hline Antibioticoprofilaxia & & $32,5 \%$ \\
\hline Sim & 114 & $13,7 \%$ \\
Não realizado & 48 & $53,8 \%$ \\
Não informado & 189 & \\
\hline Antibiótico de escolha para antibioticoprofilaxia & & $59,6 \%$ \\
\hline Cefalotina & 68 & $40,3 \%$ \\
\hline
\end{tabular}

Fonte: Marinho MPSM e Soeiro CMO, 2021.

A prevalência de infecção puerperal no parto cesáreo foi de 95,2\% ( $n=361$ ), ou seja, maior que a do parto normal sendo esta 4,8\% ( $n=19)$. Entre os tipos de infecção puerperal, neste estudo predominou a Infecção de Sítio Cirúrgico (ISC) caracterizando $95,2 \%$ da amostra ( $n=334$ ). Em segundo lugar tem-se Mastite com 4,3\% $(n=15)$, seguida de Endometrite e Peritonite ambos constituindo $0,6 \%(n=2)$ da amostra cada um. 
Os principais critérios clínicos associados à infecção do sítio cirúrgico foram: febre, presente em 43,5\% dos casos ( $n=153)$, seguido pela hiperemia, relatado em $35,0 \%(n=123)$. Outros critérios clínicos frequentes foram a secreção purulenta, em 28,2\% ( $n=99)$ e a secreção serossanguinolenta, em 24,5\% dos casos ( $n=86)$.

A ultrassonografia abdominal foi realizada em $53,8 \%(n=189)$ das pacientes. Dessas $51,8 \%(n=98)$ destes exames evidenciaram abscesso de parede e $24,8 \%(n=47)$ hematoma, além de $8,9 \%(n=17)$ sem alterações, seguidos pela combinação de hematoma + abscesso representando $5,8 \%,(n=11)$.

Todas as 351 pacientes receberam antibioticoterapia neste estudo, sendo, pois, todas em regime de internação. Os esquemas mais utilizados foram os tríplices, uma cefalosporina ou penicilina associada à Gentamicina e Metronidazol, destes a tríade Cefalotina associada à Gentamicina e Metronidazol foi dominante com uso em $53,8 \%$ dos casos $(n=189)$. O segundo esquema mais utilizado foi Ceftriaxona associada à Gentamicina e Metronidazol, em $17,7 \%$ dos casos ( $n=62)$. O terceiro esquema mais utilizado foi a Cefalotina associada ao metronidazol, em $13,1 \%$ dos casos $(n=46)$, seguida dos esquemas de Ceftriaxona associada à Clindamicina e Gentamicina e Ceftriaxona em monoterapia, conforme Tabela 4.

Tabela 4 - Perfil do uso de antibióticos no tratamento da infecção puerperal.

\begin{tabular}{lcc}
\hline Antibióticos & Frequência de escolha (N) & $\%$ \\
\hline Ampicilina + Gentamicina + Metronidazol & 2 & $0,60 \%$ \\
Cefalotina + Gentamicina + Metronidazol & 189 & $53,80 \%$ \\
Cefalotina + Metronidazol & 46 & $13,10 \%$ \\
Cefepime & 2 & $0,60 \%$ \\
Ceftriaxona & 11 & $3,10 \%$ \\
Ceftriaxona+ Metronidazol & 3 & $0,90 \%$ \\
Ceftriaxona +Clindamicina + Gentamicina & 36 & $10,30 \%$ \\
Ceftriaxona + Gentamicina + Metronidazol & 62 & $17,70 \%$ \\
\hline
\end{tabular}

Fonte: Marinho MPSM e Soeiro CMO, 2021.

Em relação ao tempo médio de antibioticoterapia, a grande maioria das pacientes: $86,3 \%,(n=303)$ receberam o antibiótico dentro do intervalo de 7-10 dias, e apenas uma pequena parte representada por 8,5\% $(n=30)$ recebeu antibiótico por mais de 10 dias, e 5,1\% $(n=18)$ recebeu por menos de 7 dias.

Sobre a coleta de fragmento da ferida operatória para realização de cultura, tal procedimento não foi realizado em nenhuma das pacientes participantes do estudo. Em relação à drenagem abdominal, 4,5\% $(n=16)$ das puérperas foram submetidas a este procedimento, sendo que na maioria $(3,1 \%, n=11)$ drenou-se abscesso e o restante, $1,4 \%(n=5)$, hematoma. Por fim, o procedimento de ressutura foi realizado em menos da metade das pacientes, isto é, $33,1 \%(n=116)$.

A maioria das pacientes obtiveram êxito em sua evolução clínica, pois lançou-se mão de todas as ferramentas disponíveis para o sucesso no seguimento clínico, sendo elas antibioticoterapia, abordagem cirúrgica, acompanhamento em UTI e etc. E, portanto, 99,4\% $(n=349)$ receberam alta por melhora do quadro geral. As restantes $(n=2)$ evadiram da maternidade de referência sendo perdido o seguimento clínico das mesmas.

\section{DISCUSSÃO}

O principal objetivo deste estudo consiste em avaliar a prevalência de infecção puerperal, além de descrever os principais fatores que corroboram para tal desfecho. Encontrou-se uma prevalência de 2,28\% de infecção puerperal dentre a amostra analisada, taxa que se assemelha as demais maternidades no nordeste brasileiro, como no estudo retrospectivo sobre prevalência de infecção puerperal em puérperas em uma maternidade pública do Maranhão realizado por Santos AA, et al. (2017) onde constatou-se elevadas taxas de infecção de sítio cirúrgico durante o período de estudo, entre 1,6\% e 2,3\%.

Em outro estudo transversal realizado por Santos VB, et al. (2017) com 855 puérperas em maternidades públicas e privadas de Alagoas, encontrou-se a prevalência de infecção puerperal de 6,8\%. Desta forma, pode-se perceber que a prevalência de Infecção Puerperal varia de acordo com os estudos, regiões e serviços analisados, mas se mantém alta na maioria deles, inclusive em nosso estudo. 
Quanto as categorias de IP, neste estudo predominou a Infecção de Sítio Cirúrgico (ISC) caracterizando 95,2\% da amostra, seguida de Mastite com 4,3\%, Endometrite e Peritonite ambos constituindo 0,6\% ( $n=2)$ da amostra cada um. Esses resultados são similares ao de Monteiro TLVA, et al. (2016), com 94,6\% do total correspondente a infecção de sítio cirúrgico, seguido pela mastite, com 4,2\%. Outro estudo realizado por Soares FAF, et al. (2018), os tipos de infecções puerperais mais frequentemente diagnosticadas foram as mastites, com $44 \%$ do total, e as infecções de ferida operatória de cesárea, representada por $26 \%$ do total. No entanto, na maioria dos estudos encontrados há predomínio das infecções de sítio cirúrgico, o que consolida os achados em nosso estudo.

Em relação à faixa etária predominante de infecção puerperal, Crizóstomo CD, et al. (2016) e Cavalcante MFA, et al. (2015) encontram o predomínio desta comorbidade na faixa de idade de 20 a 29 anos, e em mulheres entre 18 a 30 anos, respectivamente. Em consoante com a literatura vigente, este estudo apresentou um predomínio de infecção puerperal em pacientes mais jovens, na faixa de idade entre 20-24 anos $(28,4 \%$ do total).

Soares FAF, et al. (2018) apresentou em seu estudo a predominância infecção puerperal em mulheres primíparas (46\%). Ademais, Santos AA, et al. (2017) verificou em seu estudo que $60,8 \%$ das participantes que apresentaram infecção puerperal eram primigestas, sendo submetidas ao parto cesáreo. Desta forma, assim como demonstrado em trabalhos anteriores, este estudo também encontrou uma maior porcentagem de primigestas $(38,7 \%)$ dentre o total analisado.

Neste trabalho a principal comorbidade associada ao risco de infecção puerperal foi a infecção do trato urinário, presente em $37,6 \%$ das gestantes. Vários estudos confirmam a importância da infecção urinária como um fator de risco para infecção puerperal, como o estudo de Mascarello KC, et al. (2017) que identificou como principais fatores de risco para infecção puerperal, dentre outras coisas, a cesariana, ruptura prematura das membranas, imunossupressão sistêmica da gestação e infecção urinária na gestação.

Segundo a Federação Brasileira das Associações de Ginecologia e Obstetrícia (FEBRASGO) (2016), a assistência pré-natal cautelosa representa um pilar essencial para a redução da morbimortalidade maternofetal. Quando esta não é apropriada há um grande comprometimento dos indicadores de saúde maternoinfantil. Neste estudo constatou-se que dentre as $68,9 \%$ das pacientes que realizaram o pré-natal, $47,1 \%$ compareceram a menos de 6 consultas, que é o valor mínimo preconizado pelo Ministério da Saúde segundo a Portaria ํㅜ 570, de 1ํ de junho de 2000.

De acordo com o boletim № 199 de 2018 do ACOG não há diferença significativa de efetividade entre as cefalosporinas de primeira, segunda e terceira geração na profilaxia de infecção de ferida operatória, sendo ambos altamente eficazes. Desta forma, tanto a Cefazolina quanto a Cefalotina, utilizadas neste estudo, apresentam eficácia para profilaxia de Infecção de Ferida Operatória.

Entretanto, pode-se perceber que $32,5 \%$ das pacientes apresentaram infecção puerperal a despeito do uso de antibiótico profilático de classe adequada. Tal fato pode ser explicado porque outros fatores que também influenciam a eficiência da antibioticoprofilaxia não estavam informados no prontuário, como o tempo em que o antibiótico foi administrado em relação ao início do procedimento, além do tempo cirúrgico, o preparo da paciente, e, portanto, não puderam ser analisados para averiguar a correta administração da profilaxia.

De acordo com Santos VB, et al. (2017), é necessária a identificação do microrganismo causador da infecção do sítio cirúrgico, pois esta orientará a escolha da correta antibioticoterapia da paciente. No entanto, foi observado neste estudo que nenhuma paciente fez coleta de fragmento da ferida operatória para cultura, de forma que todas realizaram tratamento empírico da Infecção de Sítio Cirúrgico.

Neste estudo encontrou-se uma porcentagem de $33 \%(n=116)$ de pacientes submetidas à ressutura de parede, o que representa aproximadamente um terço destas pacientes necessitaram passar pelo estresse de outro procedimento cirúrgico, com aumento do tempo de internação hospitalar e maior ônus à instituição. No entanto, em um estudo que analisou um total de 6.764 cesarianas em uma maternidade pública houve uma alta taxa de ressutura, correspondente à $73,8 \%$ dos casos em que ocorreu Infecção de Ferida Operatória, o que demonstra que as taxas de ressutura em nosso estudo estão baixas, comparadas à outros estudos (SANTOS AA, et al., 2017). 


\section{CONCLUSÃO}

Ao longo de nosso estudo foi traçado o perfil das pacientes em estudo, o qual consiste em mulheres jovens, multíparas, portadoras de fatores de risco para IP, com foco especial para Infecção do Trato Urinário. Neste estudo a taxa de IP foi de 2,28\%, a qual assemelha-se com a média nacional, com predomínio da Infecção de Sítio Cirúrgico. As classes de antibióticos mais utilizadas para o foram as cefalosporinas de primeira e segunda geração, associadas ao aminoglicosídeos e metronidazol. O presente estudo, em que se pese suas limitações, contribuiu com o conhecimento acerca dos aspectos epidemiológicos da infecção puerperal no estado do Amazonas.

\section{REFERÊNCIAS}

1. AGÊNCIA NACIONAL DE VIGILÂNCIA SANITÁRIA (ANVISA). Medidas de Prevenção e Critérios Diagnósticos de Infecções Puerperais em Parto Vaginal e Cirurgia Cesariana. 2017. Disponível em: https://segurancadopaciente.com.br/wp-content/uploads/2018/02/Caderno_8_Anvisa.pdf. Acessado em: 12 de maio de 2021.

2. AMERICAN COLLEGE OF OBSTETRICIANS AND GYNECOLOGISTS. ACOG Practice Bulletin No. 120: Use of prophylactic antibiotics in labor and delivery. 2011; 117(6): 1472-1483.

3. AMERICAN COLLEGE OF OBSTETRICIANS AND GYNECOLOGISTS. ACOG Practice Bulletin No. 199: Use of Prophylactic Antibiotics in Labor and Delivery. 2018; 132(3): e103-e119.

4. BRASIL. Ministério da Saúde. Portaria no 570 , de $1^{\circ}$ e junho de 2000. Disponível em: https://bvsms.saude.gov.br/bvs/saudelegis/gm/2000/prt0570_01_06_2000_rep.html. Acessado em: 12 de maio de 2021.

5. CAVALCANTE MFA, et al. Caracterização das infecções puerperais em uma maternidade pública municipal de Teresina em 2013. Rev Epidemiol Control Infect., 2015; 5(10): 47-51.

6. CRIZÓSTOMO CD, et al. O perfil das mulheres com amniorrexe prematura em uma maternidade da rede pública estadual. R. Interd., 2016; 9(1): 135-142.

7. DIAS CMCC, OLIVEIRA JR. Fatores de risco da infecção puerperal: revisão bibliográfica. Arquivo de Biblioteca Atualiza, 2011.

8. FEDERAÇÃO BRASILEIRA DAS ASSOCIAÇÕES DE GINECOLOGIA E OBSTETRíCIA (FEBRASGO). Manual de Orientação Assistência ao Abortamento, Parto e Puerpério. 2010 . Disponível em: http://professor.pucgoias.edu.br/SiteDocente/admin/arquivosUpload/13162/material/ASSIST\%C3\%8ANCIA\%20AO\% 20PARTO,\%20PUERP\%C3\%89RIO\%20E\%20ABORTAMENTO\%20-\%20FEBRASGO\%202010.pdf. Acessado em: 12 de maio de 2021.

9. GONÇALVES MVC, et al. Endometriose puerperal e sepse: uma revisão. Rev Med Minas Gerais, 2012; $22(5): 21-24$.

10. MARTINS ACS, SILVA LS. Perfil epidemiológico de mortalidade materna. Revista Brasileira de Enfermagem, 2018; 71(1): 677-683.

11. MASCARELLO KC, et al. Complicações maternas e cesárea sem indicação: revisão sistemática e meta-análise. Rev. Saúde Pública, 2017; 51(105): 23-27.

12. MONTEIRO TLVA, et al. Eventos de infecção puerperal em uma maternidade de referência no município de Caxias, Maranhão. Rev. enferm. UFPI, 2016; 5(2): 11-15.

13. PRAIA IG, SILVA SM. Análise do uso de antibióticos na profilaxia de feridas operatórias nas cesarianas realizadas em uma maternidade, no período de 2015 a 2018. Revista Eletrônica Acervo Saúde, 2021; 13(2): e6223.

14. SANTOS AA, et al. Antibioticoprofilaxia em gestantes submetidas à cesariana. Rev. enfermagem UFPE online, 2017; 11(5): 1842-1846.

15. SANTOS VB, et al. Infection of surgical site in women submitted to cesarian in a public maternity. Revista de Pesquisa em Saúde, 2017; 18(1): 14-17.

16. SAY L, et. al. Global causes of maternal death: a WHO systematic analysis. The Lancet. Global health, 2014; 2(6): 323-333.

17. SOARES FAF, et al. Epidemiologia das infecções puerperais numa maternidade pública de teresina-piauí. REV. Uniabeu, 2018; 11(28): 357-366.

18. TIES B, et al. Global epidemiology of use of and disparities in caesarean sections. The Lancet, 2018; 392(1): 13411348.

19. UNIVERSIDADE FEDERAL DO RIO DE JANEIRO (UFRJ). Manual de rotinas assistenciais da maternidade-escola da Universidade Federal do Rio de Janeiro. 2018. Disponível em: http://www.me.ufrj.br/index.php/atencao-asaude/protocolos-assistenciais.html. Acessado em: 01 de junho de 2021.

20. WORLD HEALTH ORGANIZATION (WHO). World Health Organization recommendations for prevention and treatment of maternal peripartum infections. 2015. Disponível em http://apps.who.int/iris/bitstream/handle/10665/186171/9789241549363_eng.pdf;jsessionid=DDE86CD2757FF45EAE 79139DEAFBEA13?sequence=1. Acessado em: 12 de maio de 2021. 